\title{
Immobilization of the Alkaline Phosphatase on Collagen Surface via Cross-Linking Method
}

\author{
Parichehr Hanachi 1, Farzaneh Jafary ${ }^{2, *}$, Fariba Jafary ${ }^{3}$, Shima Motamedi 4 \\ ${ }^{1}$ Department of Biotecknology, Biochemistry unite, Alzahra University, Tehran, Iran \\ ${ }^{2}$ Department of Biochemistry Shahid Sadoughy University, Faculty of Medicine, Yazd, Iran \\ ${ }^{3}$ Young Researchers Club, I.A.U, Falavarjane University, Isfahane, Iran \\ ${ }^{4}$ School of Veterinary Medicine, Razi University, Kermanshah, Iran \\ ${ }^{*}$ Corresponding author: Farzaneh Jafary, Department of Biochemistry Shahid Sadoughy University, Faculty of Medicine, Yazd, Iran. Tel/Fax: $+98-$ \\ 3136544522, E-mail: fr.jafary@yahoo.com
}

Received: April 10, 2015; Revised: July 02 2015; Accepted: August 03, 2015

\begin{abstract}
Background: Collagen, the most abundant protein in the human body, and as an extracellular matrix protein, has an important role in the fiber formation. This feature of the collagen renders establishment of the structural skeleton in tissues. Regarding specific features associated with the collagen, such as, formation of the porous structure, permeability and hydrophilicity, it can also be used as a biocompatible matrix in the enzyme engineering.

Objectives: The aim of the present study was to investigate the application of the type I collagen as a matrix for alkaline phosphatase immobilization using cross-linking method.

Material and Methods: The Alkaline phosphatase was covalently immobilized on collagen matrix by using 1-ethyl-3(dimethylaminopropyl) carbodiimide hydrochloride (EDC). The source of the alkaline phosphatase was from the bovine intestinal mucous. After that, the activity of the immobilized enzyme was assayed under different experimental conditions. Results: The optimum $\mathrm{pH}$ was similar to that of the free enzyme, whereas the optimum temperature and thermal stability were shown some increments. The surface topography of the collagen matrix containing immobilized enzyme and ALP (Alkaline phosphatase) deficient was investigated by Atomic-force microscopy (AFM). Images that have been obtained applying AFM show significant differences between uncovered and immobilized enzyme- matrix surface topography.

Conclusions: Our findings suggest that type I collagen can be utilized as a matrix for alkaline phosphatase immobilization via cross-linking method.

Keywords: Alkaline phosphatase; Enzyme activity; Immobilization; Matrix
\end{abstract}

\section{Background}

One of the most essential and advantageous strategies in the field of clinical and the industrial enzyme technology is the enzymes immobilization. Indeed, in order to improve enzyme application, immobilization techniques, such as adsorption, multipoint and multi subunit covalent binding, as well as entrapment can be used. The products of the reaction which are considered as the out puts of these methods improve specific enzyme criteria, such as stability, specificity, activity, and inhibition (1).

In comparison to the free enzymes in the same condition, the immobilized enzymes have shown the desired functional characteristics, such as prolonged activity and multiple reusable capability in the industrial reactors $(2,3)$. In order to ensure the highest retention of the enzyme activity in addition tostability in the enzyme immobilization technique, there are different organic and inorganic supports, such as polymers and nanoparticles (4-6).

Collagen is among the major fibrous structural proteins which is present in the connective tissue of the vertebrates, and in particular in the bone and cartilage. Indeed, it acts as the most functional backbone of these structures $(7,8)$. Currently, more than twenty different types of the collagen have been identified with the common structural feature: the unique right-handed triple helix. The basic polypeptide unit of collagen is the repeating sequence $(G l y-X-Y)_{\mathrm{n}}$, where $X$ and $Y$ are often proline and hydroxyproline, respectively. Type I collagen is the most abundant type of collagen in the human body, especially in the bones, skins, tendons, 
vascular system, and organs (9). Collagen possesses valuable properties, such as biodegradability, biocompatibility, non-cytotoxicity, in addition to ability to support the cellular growth. In addition, the various processed products of this biomolecule such as films, sheets, beads, fibers, and sponges can be used for biomedical applications.

Collagen has particular properties including thermal stability, mechanical strength, and the ability to engage in the specific interactions with other biomolecules. The porous structure of the collagen matrix provides a large specific surface for supplying relatively high quantity of the enzyme loading in addition to high porosity giving the accessibility of the active sites along with low diffusion resistance necessary for high reaction rate and conversion $(41,42)$. The fibrouse structure of the collagen also leads to the improved catalytic efficiency of the immobilized enzymes. Therefore, the unique physical and biochemical properties of the collagen make it a suitable support for the enzyme immobilization and application in the largescale operation.

Alkaline phosphatase (Aps; EC 3.1.3.1) is a metalloenzyme (two $Z n$ and one $M g$ ) which removes phosphate groups from numerous kinds of molecules and degrades inorganic pyro phosphate $\left(p p_{i}\right)$ to inorganic phosphate (10). Alkaline phosphatase has been considered as a useful and common enzyme for different application, among which molecular biology laboratories. The reason of this selection is the possessing of the phosphate group at the $5^{\prime}$ end in DNA. Therefore, in order to prevent ligation (attachment of the $5^{\prime}$ end to the $3^{\prime}$ end in DNA molecule), terminal phosphates are removed. As a result, DNA molecules remain in linear form until the next step of the process. As well, ALP is known as a labeling enzyme which is applied in electrochemical enzyme immunoassays and immunosensors (11).

Furthermore, since ALP is essential in bone mineralization, its immobilization can be applied in bone tissue engineering.

The three common methods for immobilization of the ALP are: cross-linking, covalent binding, and adsorption. The enzyme was immobilized by crosslinking method on different matrices such as microscope glass and fibrin. The most common functional reagents that are used for cross linking the enzyme are 1-ethyl-3-(dimethylaminopropyl) carbodiimide hydrochloride (EDC), N-hydroxysuccinimide (NHS), diazo and Glycidoxypropyletrimethoxysilane (12-14). Although the ALP has been immobilized on aminated glass surface under vacuum condition without involvement of chemical reagent (15), but, collagen has not been used as matrix yet for ALP immobilization.

\section{Objectives}

The aim of the present study was to investigate if type I collagen can be used as matrix for alkaline phosphatase immobilization with cross-linking method.

\section{Materials and Methods}

\subsection{Activation of the Collagen Fiber and} Immobilization of the Enzyme

The collagen matrix (Viscofan cell carrier) was treated with $10 \mathrm{mg} / \mathrm{mL}$ of 1-ethyl-3-(dimethylaminopropyl)- carbodiimide hydrochloride (EDC) (Piers 22980) in phosphate buffer for 30 minutes (16). Activated matrix was washed with PBS and was incubated in the aqueous bovine intestinal mucosa alkaline phosphatase (3 U) (Sigma P6774) for 2 h. The collagen matrix was rinsed four times with PBS 15 min each on a shaker.

\subsection{The Alkaline Phosphatase Activity Assay}

The Taylor et al. (2006) method was used for estimation of the enzyme activity. The p-nitrophenylphosphate (pNPP) (Sigma P4744) was chosen as the substrate (15). A Stock solution of pNPP was prepared (350 $\mu \mathrm{M}$ pNPP in $25 \mathrm{mM}$ glycine at $\mathrm{pH}$ 9.5) and collagen matrix with the immobilized enzyme was treated with $2 \mathrm{~mL}$ of the substrate solution at room temperature. $90 \mu \mathrm{L}$ of $1 \mathrm{M} \mathrm{NaOH}$ and $1 \mathrm{M}$ EDTA was added every minute to the reaction mixture to stop reaction, and absorbance was determined at $405 \mathrm{~nm}$. The alkaline phosphatase activity was calculated using the methods Taylor et al. 2005 applying the following formula.

\subsection{Quantification of the Immobilized Enzyme}

The standard curve was plotted applying $2 \mathrm{~mL}$ of substrate against different concentrations of free enzyme $(3.2,6.3,12.5$ 25, 50 unit.mL-1). Subsequently, enzyme activity of ALP immobilized on collagene surface was measured in accordance to standard activity curve.

\subsection{Estimation of the Optimum $\mathrm{pH}$ of the Free and Immobilized ALP}

The alkaline phosphatase activity (both free and immobilized) was determined at $37^{\circ} \mathrm{C}$ and at different pHs $(8.5,9,9.5,10$, and 10.5$)$ with $350 \mu \mathrm{M}$ of the pNPP as substrate. 
3.5. Determination of the Optimum Temprature for Free and Immobilized ALP

The activity of the free and immobilized enzymes was detected at different temperatures ranging from $30^{\circ} \mathrm{C}$ to $50^{\circ} \mathrm{C}$ in presence of $2 \mathrm{~mL}$ of $350 \mu \mathrm{M}$ pNPP.

\subsection{Thermal Stability of the Free and Immobilized Enzyme}

The free and immobilized enzymes were incubated at $37^{\circ} \mathrm{C}$ and every 5 min the activity was determined up to $50 \mathrm{~min}$.

\subsection{Reusability of the Immobilized ALP}

The activity of 3 units of the immobilized enzyme on the matrix was calculated first.Then the matrix was washed using phosphate buffer at $\mathrm{pH}$ range 6.5-7.5.

\subsection{Storage Stability of the Immobilized ALP}

The storage condition for the collagen matrix with immobilized enzyme was determined in $P B S$ buffer with $\mathrm{pH}$ range 6.5-7.5 at room temperature for one month and the activity was assayed as a function of the time.

\section{Results}

\subsection{Properties of the Immobilized ALP}

In the presen study the optimum $\mathrm{pH}$ and temperature, thermal stability, reusability, and storage stability of the immobilized enzyme were studied. The total amount of the immobilized enzyme on a surface area of $2.25 \mathrm{~cm}^{2}$ of the synthesized collagen matrix was determined. It was found that 3 units of the enzyme has covered this unit area (Figures 1,2).

\subsection{The Optimum $p H$}

The effect of the $\mathrm{pH}$ on immobilized alkaline phosphatase activity was investigated and compared with the free enzyme (Figure 3). Result indicated that the optimal $\mathrm{pH}$ value for the immobilized and free enzyme was the same $(\approx 9.5)$. The $\mathrm{pH}$ changes in the immobilized enzyme can be associated with the polymer characteristics and product accumulation on the matrix. Moreover, observed perturbations on the $\mathrm{pH}$ profiles are likey due to the product accumulation on matrix in such a way that enzyme activity itself could be hindered.

\subsection{Optimum Temperature}

The best temperature for free enzyme activity was $35^{\circ} \mathrm{C}$ which was enhanced to $40^{\circ} \mathrm{C}$ for immobilized enzyme (Figure 4).

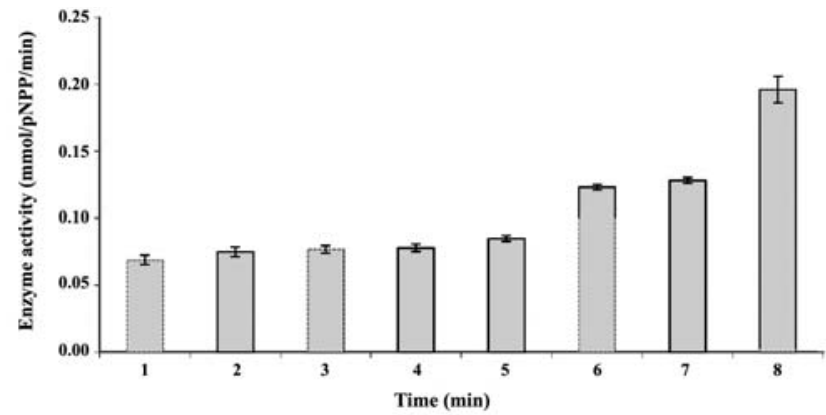

Figure 1. Assessment of the immobilized alkaline phosphatase according to the Taylor's method with p-nitrophenylphosphate as a substrate

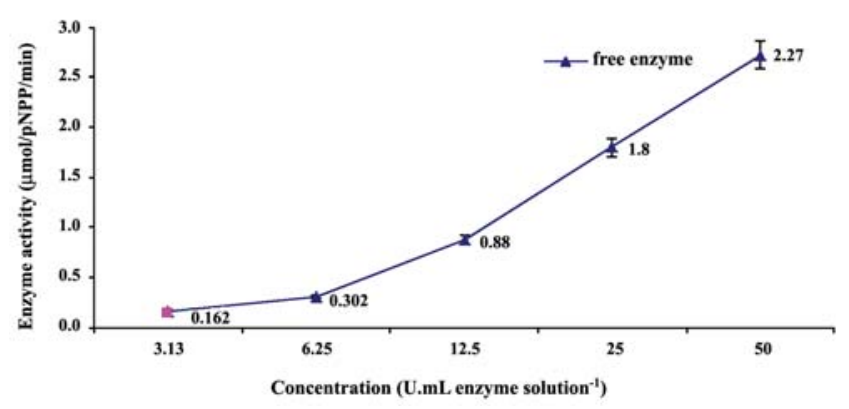

Figure 2. Standard activity curve, this curve was used to estimate the amount of the immobilized enzyme



Figure 3. The effect of $\mathrm{pH}$ on the activity of the free and immobilized enzymes. Both enzymes show the highest activity at $\mathrm{pH} 9.5$ although the immobilized enzyme shows higher activity for a wider range of $\mathrm{pHs}$

\subsection{Thermal Stability}

The thermal stability of the free and the immobilized enzymes on the collagen matrix was determined. According to the plots that are shown in the figure Figure 5, the immobilized ALP displays a rise in the thermal activity in comparison to the free enzyme. The covalent immobilization of the alkaline phosphatase increased denaturation resistance which was probably 


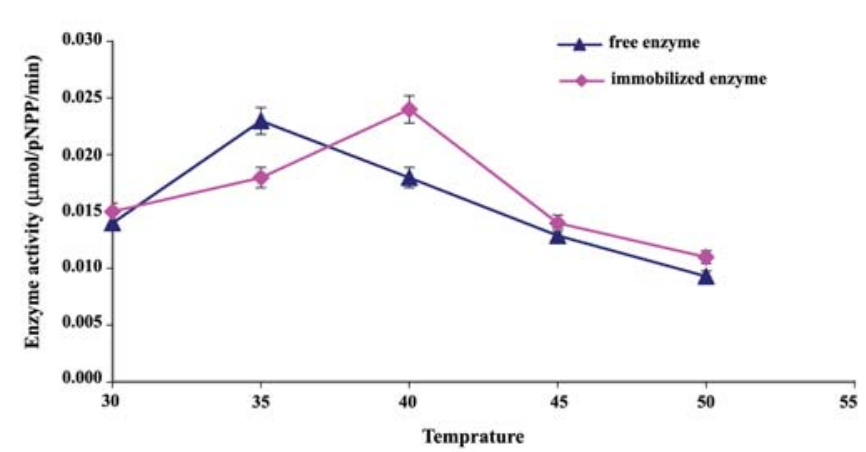

Figure 4. The effect of temperature $\left(30-50^{\circ} \mathrm{C}\right)$ on the enzyme activity of the free and immobilized enzymes. The immobilized enzyme shows an optimal activity at a higher temperature $\left(40^{\circ} \mathrm{C}\right)$

a consequence of the multipoint attachment acquired in the immobilization process.

\subsection{Reusability}

Reusability of the immobilized enzymes has been considered a valuable property in the enzyme engineering. Our results indicated that alkaline phosphatase activity was remained about $48.6 \%$ after 9 times (Figure 6).

\subsection{Storage Stability and AFM Characterization of the Collagen}

The immobilized enzyme was stored at the room temperature for one month and the activity was assayed during this period. It was found that the residual enzyme activity is approximately $40 \%$ after one month of the storage. The surface topography was investigated by applying AFM on collagen matrix containing the immobilized ALP and the matrix without

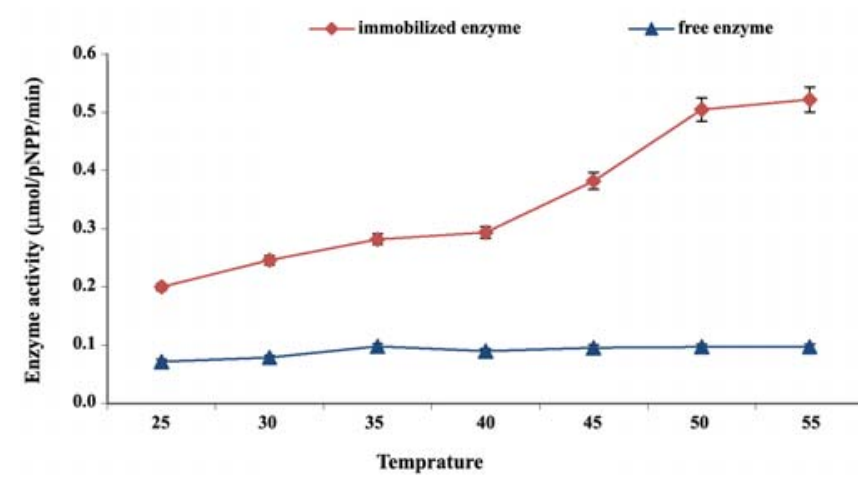

Figure 5. Thermal stability of the free and the immobilized enzyme after $50 \mathrm{~min}$ of the incubation at $37^{\circ} \mathrm{C}$. Immobilization of thr ALP has resulted in an increased thermal stability



Figure 6. The effect of the repeated use $(\mathrm{N})$ on the activity of the immobilized enzyme. The immobilized enzyme keeps around $50 \%$ of the enzymatic activity upon 9 times of the application

ALP as shown in Figure 7. The images demonstrated significant differences between bare and immobilized enzyme matrix surfaces topography, which revealed that the process of enzyme immobilization was successfully performed.

\section{Discussion}

Up to now, different kinds of enzymes have been immobilized on collagen membrane with either of the three methods including impregnation, entrapment, and electrolytic co deposition (12-14). However, ALP immobilization on collagen as a matrix has not been carried out yet. Vissink has immobilized heparin on collagen by using EDC and NHS in 2002 (16) and Yeminiet et al. (2006) have utilized collagen-like peptide as a matrix for immobilization of the horseradish peroxidase (17). Therefore, in the present study we have selected collagen for ALP immobilization. We selected collagen as it is mechanically stable with high tensile strength and could be altered into different sizes and shapes by various physical and chemical modifications. On the other hand, collagen has suitable functional groups such as carboxyl groups on glutamates and amine groups on lysines, and arginine amino acids that can participate in cross-linking reaction. These advantages make collagen suitable for biomedical and biomaterial applications.

In this study, EDC was used as the cross linking reagent, the most common form of 1-ethyl-3-(3dimethylaminopropyl)- carbodiimide, and a water soluble hydrochloride derivative of the carbodiimide. The optimum $\mathrm{pH}$ ranges for this compound are 4.0-6.0 . The goal for using this compound as a carboxyl activating agent was the coupling of the primary amines to yield amide bond. Indeed the EDC can be coupled 



Figure 7. The AFM images of the collagen matrix surface, before (left) and after immobilization (right). The coarse surface was decreased following to the bindingwith the enzyme

with carboxyl groups of Glutamates or Aspartat, and amino group of lysine or hydroxylysine residues. The most important properties of EDC include lack of cytotoxicity and slow enzymatic degradation which make it as a suitable candidate for in vivo studies in living systems. Furthermore, EDC increases intra and inter helical linkages in the tropocollagen molecules without an intermicrofibrillar crosslinks. These properties makes it suitable for enzyme immobilization, because no major change could occur in the enzyme structure during the process of the immobilization.

Moreover, enzyme structural alterations during the process of immobilization and formation of binding between enzyme and matrix, must be kept minimized. Therefore, providing information regarding immobilized enzymatic properties is considered as an important step in this context. The reason for this importance is its application in the immobilization of the enzymes and illustration of the relationships between structure-function and the mechanism of enzyme reaction. Therefore, in this study the optimal $\mathrm{pH}$ and temperature, thermal stability, reusability, and storage stability of the immobilized enzyme were studied.

According to the earlier studies, the optimum $\mathrm{pH}$ for hydrolysis is variable based on the nature and concentration of the substrate (18). Therefore, optimum $\mathrm{pH}$ for hydrolysis of the selected substrate at certain concentrations can be compared for alkaline phosphatases which are obtained from different sources. The effect of the $\mathrm{pH}$ on immobilized alkaline phosphatase activity was investigated using free enzyme and comparing its activity with the immobilized enzyme. The result have indicated that the optimal $\mathrm{pH}$ value for immobilized and free enzyme are quite similar $(\approx 9.5)$ which is in agreement with the previous reports $(19,20)$. Interestingly, the immobilized enzyme showed higher activity in $\mathrm{pH} 10$ compared to the native enzyme which might be attributed to the effect of the $\mathrm{pH}$ on electrostatic interactions of the collagen (21).

The optimal temperature for the free enzyme activity was found to be $35^{\circ} \mathrm{C}$ which shifted to $40^{\circ} \mathrm{C}$ for the immobilized enzyme. Such significant increase could be primarily as a result of the enzyme stabilization, particularly the structural stabilization, that has occurred during the immobilization process (22).

The thermal stability of the immobilized enzyme is one of the most important criteria for application. The immobilized enzymes, particularly in the covalently bound systems are known to be more resistant to heat and denaturing agents compared to the soluble forms (23). The thermal stability of the free and the immobilized enzyme on the collagen matrix were determined. The immobilized ALP showed a rise in the thermal activity in comparison to the free enzyme. The covalent immobilization of the alkaline phosphatase has resulted in an increased denaturation resistance which was a probable consequence of the multipoint attachment that was occurred during the immobilization process.

The reusability of the immobilized enzyme is an extremely valuable asset in the enzyme engineering. Our results have indicated that alkaline phosphatase activity remained approximately $48.6 \%$ after 9 times of application, which is lower than the ALP reusability immobilized on other matrices $(24,25)$. 
The immobilized enzyme was stored at the ambient temperature for one month and the activity was measured. We found that the residual activity was still $40 \%$ even after a month elapsed.

The surface topography of the collagen matrix containing immobilized enzyme and without ALP was investigated using Atomic-force microscopy. AFM is a high-resolution type of scanning probe microscopy which makes it possible to measure the roughness of a sample surface at a high resolution. The obtained images have shown significant differences between uncovered and immobilized enzyme matrices' surface topography which suggest the successful enzyme immobilization performance. Similar studies were conducted by Joshi et al. (2007) to characterize immobilized antibody molecules on SU-8 (26) as well.

\section{Conclusions}

The out put of the results from the present study conclude that type I collagen could be introduced as a possible matrix for alkaline phosphatase immobilization applying cross-linking method. Compared to the free enzyme, some physiochemical properties of the ALP could be improved but it should be mentioned that at present collagen may not be a suitable matrix for industrial applications as the harsh conditions associated with the industry. Regarding to the consideration of the collagen as a protein compound and an ideal matrix for tissue engineering, we plan to use collagen in the future researches on cell differentiation studies.

\section{Acknowledgments}

Authors wish to acknowledge all contributions of the "Iran National Science Foundation" for funding (grant number 90004378) the present research, development, and accomplishment.

\section{References}

1. Betancor L, Lopez G.F, Hidalgo A, Alonso MN, Dellamora O, Cesar MG, Fernadez LR, Guisan J. Different mechanisms of protein immobilization on glutaraldehyde activated supports: effect of support activation and immobilization conditions. EMT. 2006;39: 877-882.

2. Mateo C, Palomo JM, Fernandez-Lorente G, Guisan JM, Fernandez-Lafuente R. Improvement of enzyme activity, stability and selectivity via immobilization techniques. EMT. 2007; 40:1451-1463.

3. Fernandes KF, Lima CS, Pinho H, Collins CH. Immobilization of horseradish peroxidase onto polyaniline polymer. Process Biochemistry. 2003;38:1379-138.

4. Fernandes KF, Lima CS, Pinho H, Collins CH. Properties of horseradish peroxidase immobilized onto polyaniline. Process Biochemistry. 2004;39:957-962.
5. Gerard M, Chaubey A, Malhotra BD. Application of conducting polymers to biosensors. Biosens and Bioelectronics. 2002;173:45-59.

6. Gomez JL, Bodalo A, Gomez E, Bastida J, Hidalgo AM , Gomez M. Immobilization of peroxidase on glass beads: An improved alternative for phenol removal. EMT. 2006;36: 1016-1022.

7. Yamaguchi Y, Mann DM, Ruoslathi E. Negative regulation of transforming growth factor- $\beta$ by the proteoglycan decorin. Nature 1990; 346: 281-284.

8. Hay ED. Extracellular matrix. J Cell Biol. 1981;91:205-223.

9. Hoshi K, Kemmotsu S, Takeuchi Y, Amizuka N, Ozawa H. The primary calcification in bones follows removal of decorin and fusion of collagen fibrils. $J B M R$. 1999;14:273-280.

10. Millan J. Alkaline phosphatase: structure, substrate specificity and functional relatedness to other members of a large superfamily of enzyme. Purin Signal. 2005;2:335-341.

11. Montornes AE, Osullivan JM. Reagentless detection of alkaline phosphatase using electrochemically grafted films of aromatic diazonium salts. J Electronal Chem. 2006;587:140-147.

12. Osathanon T, Giachelli C, Somerman M. Immobilization of alkaline phosphatase on microporousnanofibrous fibrin scaffolds for bone tissue engineering. Biomaterials. 2009;30: 4513-4521.

13. Sharmin F, Rakshit S, Jayasuriya H. Enzyme immobilization on glass surfaces for the development of Phosphate detection Biosensors. CIGR. 2007;9:6-19.

14. Ehlert N, Muller P, Stieve M, Behrens P. Immobiliation of alkaline phosphatase on modified silica coating. Micropor Mesopor Mat. 2010;131:51-57.

15. Taylor R, Fournier S, Simons B, Kaplan H, Hefford M. Covalent protein immobilization on glass surfaces: Application to alkaline phosphatase. $J$ Biotechnol. 2005;118:265-269.

16. Wissink M, Beernink R, Pieper JS, Poot AA, Engbers G, Beugeling T, Aken WJ, Feijen J. Immobilization of heparin to EDC/NHS-crosslinked collagen. Characterization and in vitro evalution. Biomaterials. 2001;22:151-163.

17. Yemini M, Kaplan D, Rishpon J. Collagen-like peptide as a matrix for enzyme immobilization in electrochemical biosensors. Electroanalysis. 2006;21:2049-2054.

18. Morton RK. Some properties of alkaline phosphatase of cow's milk and calf intestinal mucosa. Biochem J. 1955;60:573-582.

19. Zaborsky O. Immobilized Enzymes. Mol Nutr. 1973;19:512513.

20. Grasset L, Cordier D, Couturier R, Ville A. Immobolization of alkaline phosphatase on silk using diazo, adsorption, glutaraldehyde, and azide method: Optimum $\mathrm{pH}$ and properties of the conjugates. Biotechnocal Bioeng. 1983;25:1423-1434.

21. Yuping LI, .Asadi A, Monroe M, Douglas E. pH effects on collagen fibrillogenesis in vitro. Electrostatic interactions and phosphate binding. Mat Sci Eng. 2009;29:1643-1649.

22. Emi S, Murase Y, Hayashi T, Nakajima A. Protease immobilization onto copoly (ethylene/acrylic acid) fiber. J App Polym Sci. 1990;41:2753-2767.

23. Ulbrich R, Schellenberger A, Damerau W. Studies on the thermal inactivation of immobilized enzymes. Biotechnol Bioeng. 1986;28:511-522. 
24. Chen C, Vander does L, Bantjes A. Investigations on Vinylene vinylene Carbonatecarbonate V. Immobilization immobilization of Alkaline Phosphatase onto LDPE Films Cografted with Vinylene Carbonate and A/-Vinyl-N-methylacetamide. $J$ Appl Polym Sci. 1993;47:25-36.

25. Chen C, Vander does L, Bantjes A. Investigations on Vinylene vinylene Carbonatecarbonate. VI. Immobilization immobilization of Alkaline alkaline Phosphatase phosphatase onto Poly poly (vinylene carbonate) -Jeffamine Hydrogel hydrogel Beadsbeads. J Appl Polym Sci. 1993;48:1189-1198.

26. Joshi M, Pinto R, Rao V, Mukherji S. Silanization and antibody immobilization on SU-8. Appl Surf Sci. 2007;253:31273132 . 